\title{
DPTV-based analysis of the flow-structure/wall-shear interplay in open wet clutches
}

\author{
Robin Leister $^{1 *}$, Jochen Kriegseis ${ }^{1}$ \\ ${ }^{1}$ Institute of Fluids Mechanics (ISTM), Karlsruhe Institute of Technology (KIT), Germany \\ *robin.leister@kit.edu
}

\section{Extended Abstract}

The trend to lower energy consumption in the automotive industry still offers potential in various fields of application. One powerful saving strategy is described by the idling behavior of wet clutches, where the speed difference between drive and output, and the cooling oil in combination with a sub-millimeter spacing leads to significant amounts of wall shear stress (WSS) and accordingly drag torque. Minimization of this adverse effect has been found to be possible by means of grooved clutch-disk geometries, which have been demonstrated to correlate with the drag torque (see e.g. Neupert et al., 2018). The main interplay between torque and fluid flow in open wet clutches has been analyzed by Leister et al. (2020) in a dimensionless way. Today, a detailed investigation of a clutch flow, however, is missing for a larger variety of groove patterns and the cause-effect relations remain yet to be fully understood. Especially, the clear identification of the so-called foot print of a particular groove geometry in the flow field and corresponding WSS - thus drag-torque predictions - still requires further research efforts.

To advance beyond the level of drag-torque measurements (at most) in combination with qualitative flow visualization, the method of defocusing particle tracking velocimetry (DPTV) in combination with an in-situ calibration approach (Fuchs et al., 2016) has been successfully adapted to open wet clutches by Leister et al. (2021). The chosen experimental setup and DPTV working principle are sketched in Figure 1. Particularly, the achieved spatial resolution of $12 \mu \mathrm{m}$ provides reasonably well resolved 3D3C flow information in the rotor-stator gap and, moreover, inside the grooves under investigation.

In continuation of the earlier study by Leister et al. (2021), which focuses on the fluid mechanic insights, the present study now additionally explicitly addresses the capabilities and limitations of the used DPTV

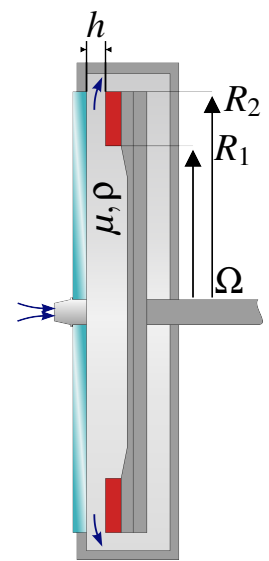

(a) test rig with relevant parameters

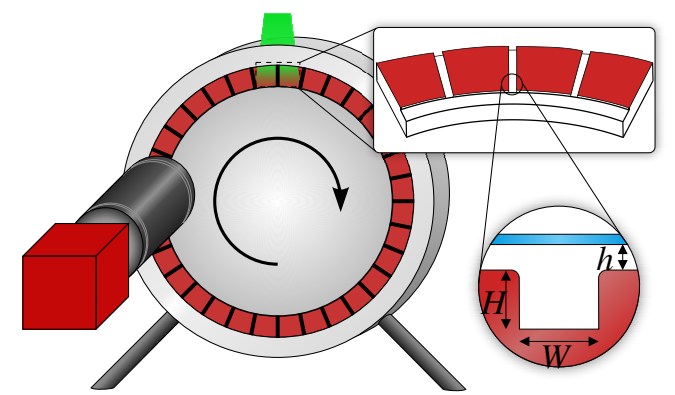

(b) camera setup (left) and open clutch test rig with groove parameters (right)

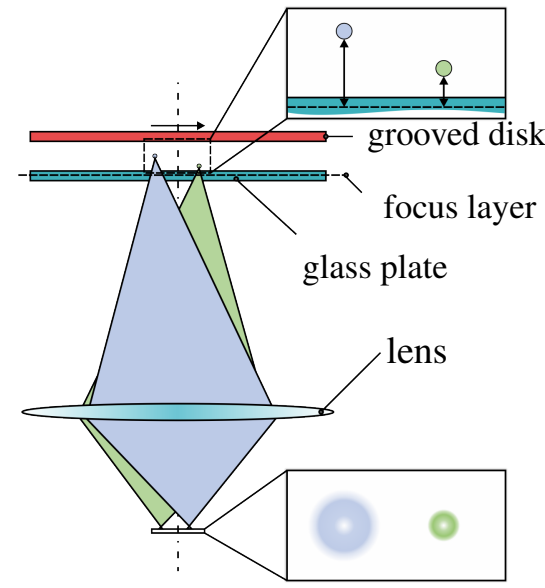

(c) Sketch of the DPTV working principle in the rotor-stator gap

Figure 1: Detailed sketches of (a) the test rig, (b) the set-up and typical groove geometries, (c) the measurement principle. 

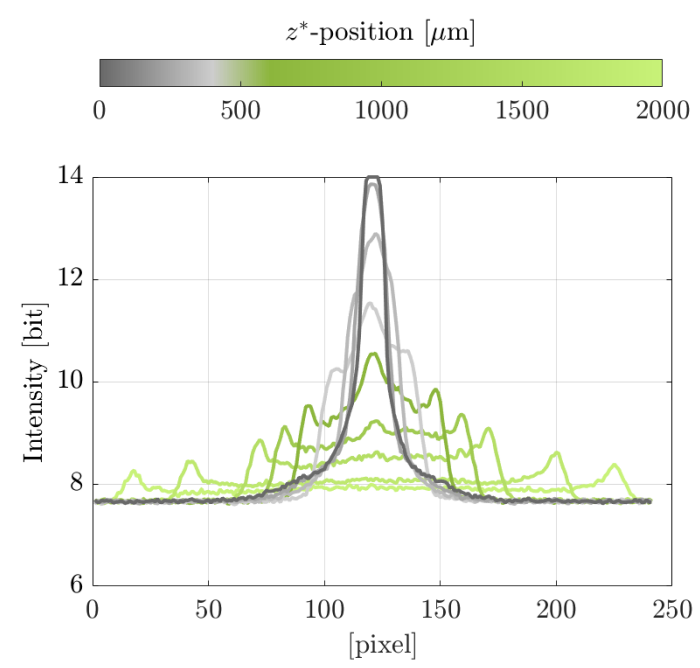

(a) Defocused diameter distribution and corresponding intensity of one particle averaged in circumferential direction. The data is acquired with a back-illuminated $10 \mu \mathrm{m}$ pinhole, which mimics a particle.

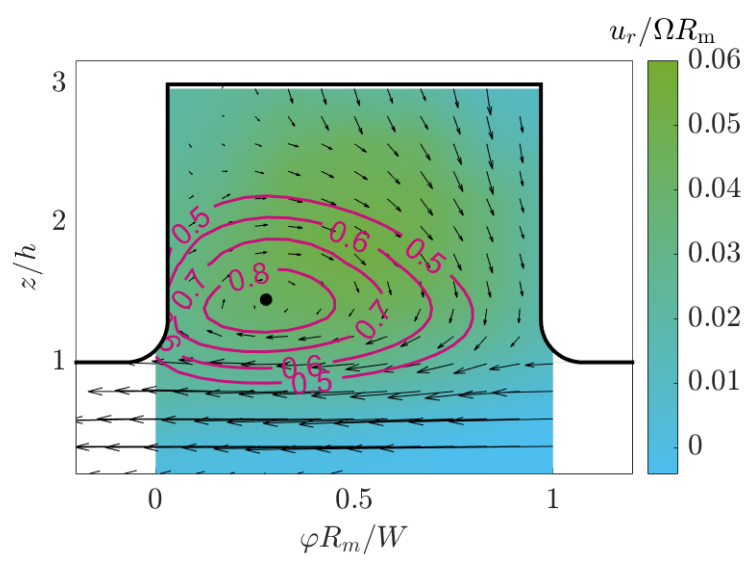

(b) Cavity roller in the rotor-fixed frame of reference. Contours of radial velocity $u_{r}$ are superimposed by $\Gamma_{1}$-isolines ( - ) and vectors of $u_{\varphi}-\Omega R_{\mathrm{m}}$ and $u_{z} .(\bullet)$ indicates the $\Gamma_{1}$ vortex-center location.

Figure 2: Example results for a radial groove of width $W=1.35 \mathrm{~mm}$ and height $H=0.97 \mathrm{~mm}$

approach as well as the conducted post-processing steps and the resulting changes upon their variation. Since for this scale of application, where the magnification is relatively moderate, compared to microscopic scenarios, the use of a normal PIV set-up, instead of a microscope, offers a large variety of possibilities and adjustment options. Both used detection methods are evaluated according their suitability and robustness for this scenario of application. The acquisition related interplay between magnification and defocusing sensibility, where the trade off between aperture and object distance must be considered, is analyzed both theoretically and explicitly for this scenario and recommendations regarding further choice of equipment are given. Figure 2(a) shows the diameter of the defocused image and the corresponding intensity. The particle image ranges from a Gaussian shape at $z^{*}=0$ to a defocused ring with low intensity $2 \mathrm{~mm}$ apart the focus layer.

To complement the study, 3D3C velocity information from the measurements are discussed according to both their respective measurement accuracy and corresponding insights for a deeper fluid mechanic understanding of the flow. Figure 2(b) shows all three velocity components for a radial groove, where a cavity roller manipulates the wall-shear stress in the vicinity of the groove. The $\Gamma_{1}$-criterion indicates the vortex-center location, which coincidences with the maximum outflow velocity $u_{r}$. The derived insights are foreseen to provide a valuable contribution to the drag-torque predictions and accordingly design optimization strategies for future clutch optimization efforts.

\section{References}

Fuchs T, Hain R, and Kähler C (2016) In situ calibrated defocusing PTV for wall-bounded measurement volumes. Measurement Science and Technology 27:084005

Leister R, Fuchs T, Mattern P, and Kriegseis J (2021) Flow-structure identification in a radially grooved open wet clutch by means of defocusing particle tracking velocimetry. Experiments in Fluids 62:29

Leister R, Najafi AF, Gatti D, Kriegseis J, and Frohnapfel B (2020) Non-dimensional characteristics of open wet clutches for advanced drag torque and aeration predictions. Tribology International 152:106442

Neupert T, Benke E, and Bartel D (2018) Parameter study on the influence of a radial groove design on the drag torque of wet clutch discs in comparison with analytical models. Tribology International 119:809821 